\title{
Correction to: On atomic composition as identity
}

\section{Roberto Loss ${ }^{1}$}

Published online: 5 March 2020

○) Springer Nature B.V. 2020

\section{Correction to: Synthese https://doi.org/10.1007/s11229-019-02295-6}

(PWCP) and (ACP) in Sect. 5 should be reformulated as follows:

(PWCP) $\exists x \exists y(\phi x \wedge \phi y \wedge x \neq y) \rightarrow \exists Y \exists z(z S F u \phi \wedge \forall x(x \prec Y \leftrightarrow x<z))$

(ACP) $\quad \exists x \exists y(\phi x \wedge \phi y \wedge x \neq y) \rightarrow \exists Y \exists z(z S F u \phi \wedge \forall x(x \prec Y \leftrightarrow(x<z \wedge A x))$

As an effect of this, throughout the paper, the theories labelled 'CAI+(PWCP)' and 'ACAI' should be understood as assuming the following 'No Improper Plurality' principle:

(NIP) $\forall X \exists y \exists z(y \prec X \wedge z \prec X \wedge y \neq z)$

Publisher's Note Springer Nature remains neutral with regard to jurisdictional claims in published maps and institutional affiliations.

The original article can be found online at https://doi.org/10.1007/s11229-019-02295-6.

Roberto Loss

robertoloss@gmail.com

1 Philosophisches Seminar, University of Hamburg, Überseering 35, 22297 Hamburg, Germany 